\title{
Computational Study of Pattern Formation for Chemically Amplified Resists in Extreme Ultraviolet Lithography
}

\author{
Masaaki Yasuda", Masanori Koyama, Kosai Fukunari, Masamitsu \\ Shirai, Hiroaki Kawata, and Yoshihiko Hirai \\ Department of Physics and Electronics, Osaka Prefecture University, \\ 1-1 Gakuen-cho, Naka-ku, Sakai, Osaka 599-8531, Japan \\ *yasuda@pe.osakafu-u.ac.jp
}

\begin{abstract}
A computational study of the pattern formation process for chemically amplified resists in extreme ultraviolet (EUV) lithography has been performed using the stochastic approach. The initial structures of the resist molecules are formed by sequential bonding of randomly arranged monomers. The effect of EUV light exposure is introduced by activation of acid generators according to the deposited energy distribution in the resist, which is calculated by Monte Carlo simulation of photoelectron scattering. Acid diffusion and the polymer deprotection reactions during the post-exposure bake (PEB) are then simulated. Development of the resist is modeled by removal of the polymers with relatively large conversion ratio from the resist. The dependence of the resist molecular weight, EUV exposure, and PEB conditions on the pattern shapes are demonstrated by the simulation.

Keywords: Extreme ultraviolet lithography, Stochastic simulation, Acid diffusion, Polymer deprotection reaction, Line edge roughness, Critical dimension error
\end{abstract}

\section{Introduction}

Extreme ultraviolet (EUV) lithography [1,2] is an effective method for sub-10-nm scale patterning. There are various issues to realize practical use of EUV lithography in high volume production of semiconductor devices. For example, high sensitivity is required for the resist materials and the pattern shapes need to be well controlled in the formation process. Computational simulations are powerful tools to assist resist material and process design. Although various computational studies have been reported [3-8], modeling of the lithography process is complicated and the issues still need to be resolved.

When the pattern size is below several tens of nanometers, the polymer molecule behavior becomes dominant in determination of the pattern structure. Therefore, molecular-scale simulations are indispensable to analyze the pattern formation process in lithography. We recently proposed the stochastic simulation technique for ultraviolet (UV) curing in UV nanoimprint lithography [9-11]. A simulation study of the pattern formation process in electron beam lithography has also been reported $[12,13]$, in which the basic resist and process characteristics were well described by the simulation.

In the present study, we applied our stochastic simulation technique to the pattern formation process for chemically amplified resists [14,15] in EUV lithography and investigated the fundamental properties of the process. We focused on introduction of the resist molecule structure into the simulation. Although modeling of electron scattering phenomena is also important to understand the pattern formation process, we roughly estimated photoelectron scattering using a simple model.

\section{Simulation model}

The trajectories of the photoelectrons generated by EUV light exposure in the resist are calculated by Monte Carlo simulation of electron scattering. As mentioned in Section 1, the EUV light exposure is roughly estimated. The wavelength of EUV light is $13.5 \mathrm{~nm}$. The probability of photon 
absorption in a resist film at depth $z$ is expressed as

$$
p(z)=\sigma \exp (-\sigma z) \quad(1)
$$

where $\sigma$ is the total ionization cross-section. The position of photoelectron production is calculated according to Eq. (1). The angular distribution of the emitted photoelectron is calculated by the following equation [16]:

$$
d p(\theta)=\frac{3}{4} \sin ^{3} \theta d \theta \quad(2)
$$

The scattering angle of the photoelectron is determined by the screened Rutherford crosssection. The energy loss of the photoelectron is calculated in inverse proportion to the square root of its energy. Although the secondary electron plays an important role in determining the exposed region, secondary electron generation was not included in the present study. The deposited energy distribution in the resist is obtained by the photoelectron trajectory calculation.

Schematic illustrations of the stochastic models of acid diffusion and polymer deprotection are shown in Fig. 1. In this study, we modeled an alkali-soluble positive-type resist. An illustration of the initial resist structure is shown in Fig. 1(a). The resist contains protected monomer and acid generator molecules. All of the molecules are expressed as a unit particle. Each monomer and acid generator is a virtual molecule. The monomers are randomly placed in space. Their positions were relaxed and optimized by using a molecular mechanics method using a Lennard-Jones potential before the polymer chain formation. The polymer chains are formed from several selected monomers. The selected monomer forms a chain with another monomer within the critical radius. The chained monomer sequentially forms another chain until the degree of polymerization reaches the set value.
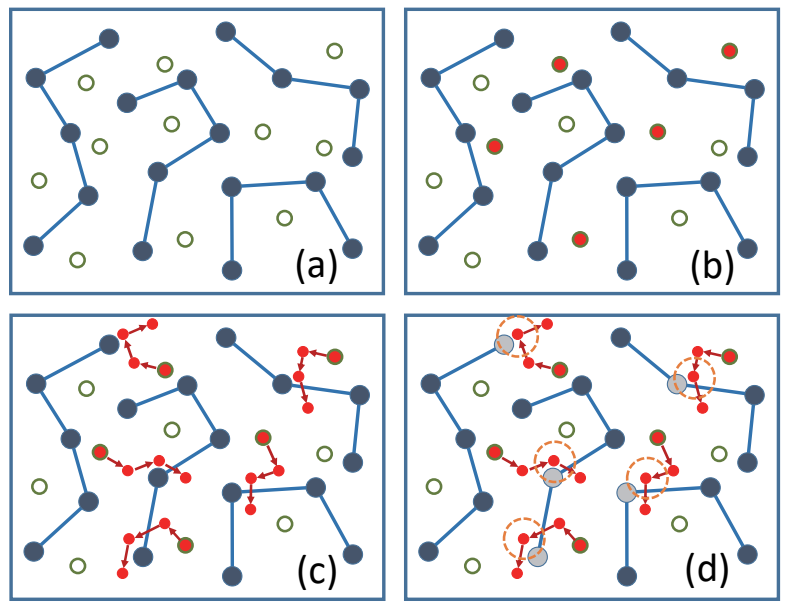

Protected monomer $\bigcirc$ Deprotected monomer Acid - Acid generator Activated acid generator Reaction radius

Fig. 1. Schematic illustration of the acid diffusion and polymer deprotection model.
The effect of EUV light exposure on the chemically amplified resists was introduced by activation of the acid generator (Fig. 1(b)). Activation of the acid generator in the resist was randomly determined in proportion to the deposited energy distribution calculated by the Monte Carlo simulation of photoelectron scattering.

The models of acid diffusion in the resist and deprotection of the monomer during the postexposure bake (PEB) process are shown in Figs. 1(c) and (d). We assume that the acid diffusion process is Brownian motion. Acid diffusion is introduced by the random-walk model with a step length according to the Gaussian probability distribution. The initial positions of the acid molecules are the same as those of the activated acid generators. The acid molecules randomly diffuse at each step. The standard deviation of the diffusion step length is set to $0.1 \mathrm{~nm}$. At each step of the random walk, when the nearest neighbor monomer is in the critical reaction radius, the acid reacts with the monomer and deprotection occurs. The critical radius for deprotection is set to $0.5 \mathrm{~nm}$. In the present simulations, quenchers were not introduced. Therefore, acid diffusion and the polymer deprotection reaction continue until the PEB simulation stops.

In the development simulation, the resist polymers with conversion ratios greater than 0.5 are removed from the resist structure. Here, the conversion ratio is defined as the ratio of the number of deprotected monomers to the number of all monomers. By removal of the polymer chains, some parts of the resist depart from the part fixed to the substrate. These floating parts of the resist are also removed in the development simulation.

\section{Results and Discussion}

\subsection{EUV light exposure}

The trajectories of the photoelectrons in the resist emitted by EUV light exposure obtained by the simulation are shown in Fig. 2. The dose distribution is assumed to be a delta function. The composition of the elements and density of the resist for poly(methyl methacrylate) (PMMA) were used to calculate of the photoelectron trajectories. Although PMMA is not a chemically amplified resist, the electron range in the resist does not significantly differ. In the present simulation, the range of photoelectrons in the resist is around $25 \mathrm{~nm}$. This value is slightly smaller than that previously reported [3,4]. As mentioned in Section 2, secondary electron generation and scattering were not introduced in the simulation. 


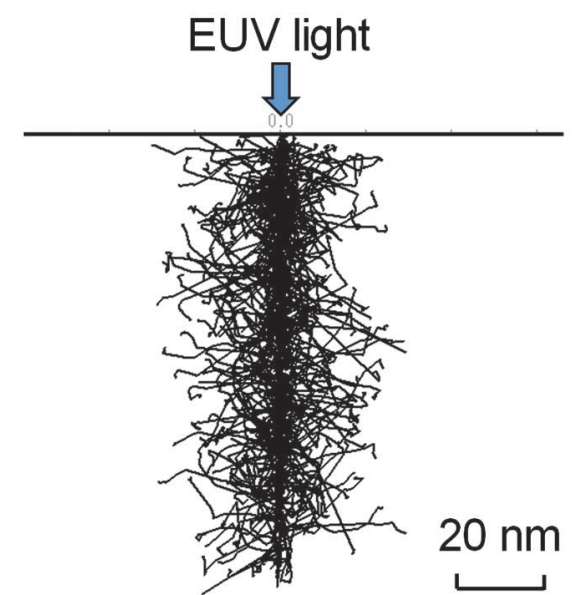

Fig. 2. Trajectories of the photoelectrons in the resist emitted by EUV light exposure obtained by Monte Carlo simulation.

The deposited energy distribution in the resist for a line pattern was calculated by a Monte Carlo simulation of photoelectron scattering to simulate the pattern formation process in EUV lithography. The deposited energy distribution for a 14-nm-wide line pattern in the resist on a Si substrate is shown in Fig. 3. The resist film thickness is $20 \mathrm{~nm}$. Because a constant dose distribution for EUV light is assumed and the convergent angle of the light is not considered, the distribution has steep edges.

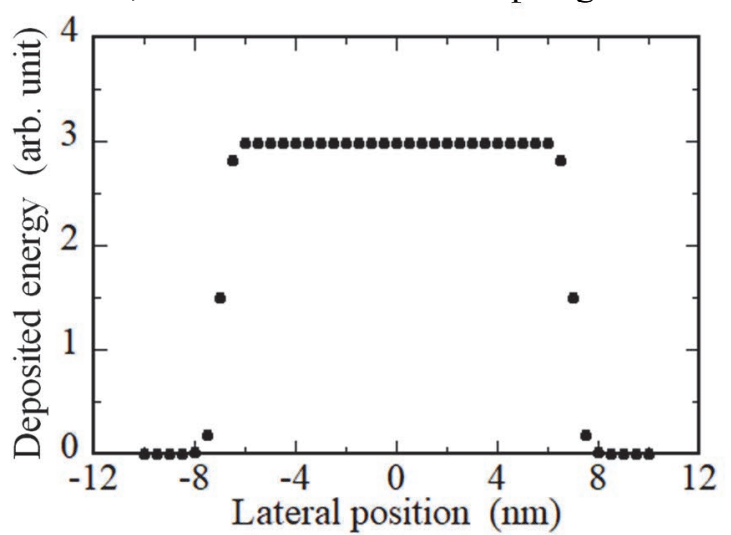

Fig. 3. Deposited energy distribution for a 14-nm-wide line pattern in the resist on a Si substrate.

\subsection{Pattern formation process}

Based on the deposited energy shown in Fig. 3, the resist pattern formation was simulated by the present model. Cross-sectional views of the acid positions in the 20-nm-thick resist for a 14-nm-wide line pattern at various PEB step numbers are shown in Fig. 4. Based on the acid diffusion coefficient obtained by experiments $\left(1.0-100 \mathrm{~nm}^{2} \mathrm{~s}^{-1}\right)[6,17]$, a PEB step number of 1 corresponds to about $0.017-$ $1.7 \mathrm{~ms}$, as estimated in our previous study [13]. The acid molecules more widely diffuse with increasing PEB step number.
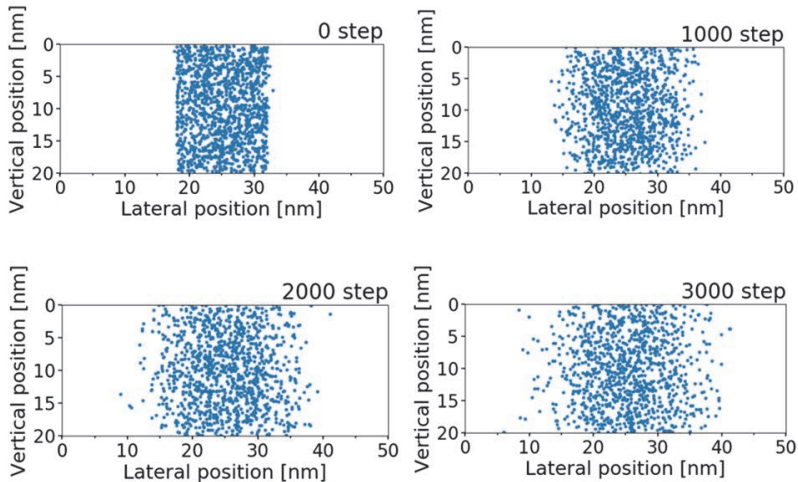

Fig. 4. Cross-sectional views of the acid positions for a 14-nm-wide line pattern exposed to EUV light at various PEB step numbers.

With the progress of acid diffusion during the PEB process, the polymer deprotection reaction also proceeds. Cross-sectional views of the conversion ratio distribution of the resist for a 14-nm-wide line pattern at various PEB step numbers are shown in Fig. 5. The region with high conversion ratio also broadens with increasing PEB step number.
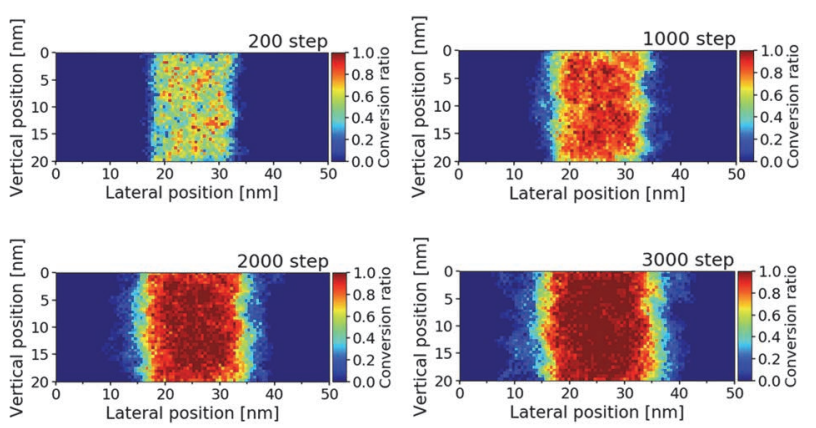

Fig. 5. Cross-sectional views of the conversion ratio distribution of the resist for a 14-nm-wide line pattern exposed to EUV light at various PEB step numbers.

By removing the resist polymers with conversion ratios greater than 0.5 from the resist structure, the developed resist pattern is obtained. An example of a 14-nm-wide line pattern on a $\mathrm{Si}$ substrate exposed to EUV light is shown in Fig. 6. The resist film thickness is $20 \mathrm{~nm}$. The mean molecular weight of the resist $M_{\mathrm{n}}=8300$. Acid diffusion and the deprotection reaction stop at PEB step number 3000 . Each polymer chain is shown in a different color.

A relatively fine pattern is obtained at this condition. The developed pattern width is about 13 $\mathrm{nm}$, which is slightly narrower than the design size. Although the sidewalls of the pattern are vertical, the pattern edges are rough. Several polymer chains at the edge stick out of the resist. The line edge roughness (LER) of this pattern is estimated to be about $4 \mathrm{~nm}$. 


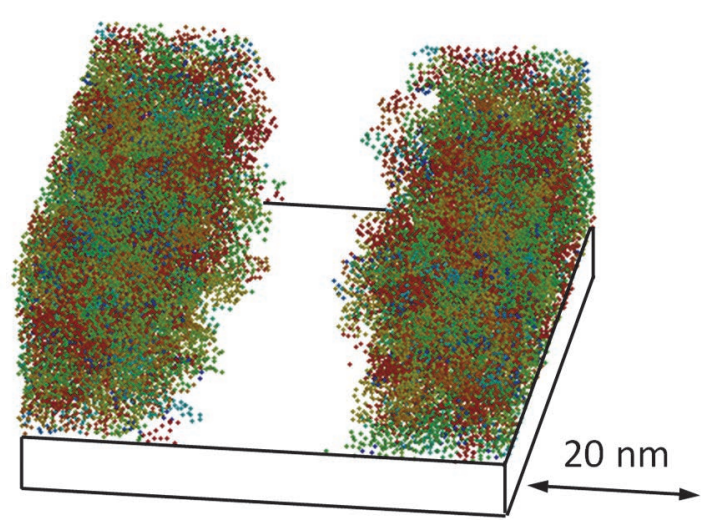

Fig. 6. Bird's-eye view of the 14-nm-wide line pattern in the resist exposed to EUV light.

\subsection{Pattern evaluation}

We then evaluated the 14-nm-wide line pattern obtained by the simulation. The LER as a function of $M_{\mathrm{n}}$ for three different EUV light doses is shown in Fig. 7. The LER was calculated from the pattern shapes for PEB step number 5000. In the present simulation, the resist is a virtual resist. Therefore, we express the dose as the relative value. Activation of the acid generator depends on the energy deposited on the resist. We define the case where all of the acid generators are activated in the maximum deposited energy region of the resist as a relative dose of 1.0. The other doses are defined as the ratio of the number of activated acid generators to that of the relative dose of 1.0.

The LER increases with increasing $M_{\mathrm{n}}$ for all three EUV light doses. The larger polymer molecules are removed during the development process for the resist with larger $M_{\mathrm{n}}$. This causes larger LER for larger $M_{\mathrm{n}}$. The LER also increases with decreasing relative dose. When the dose is small, the number of generated acid molecules is small. This causes fluctuation of the polymer deprotection reaction and results in a large LER. This trend is more pronounced for smaller $M_{\mathrm{n}}$.

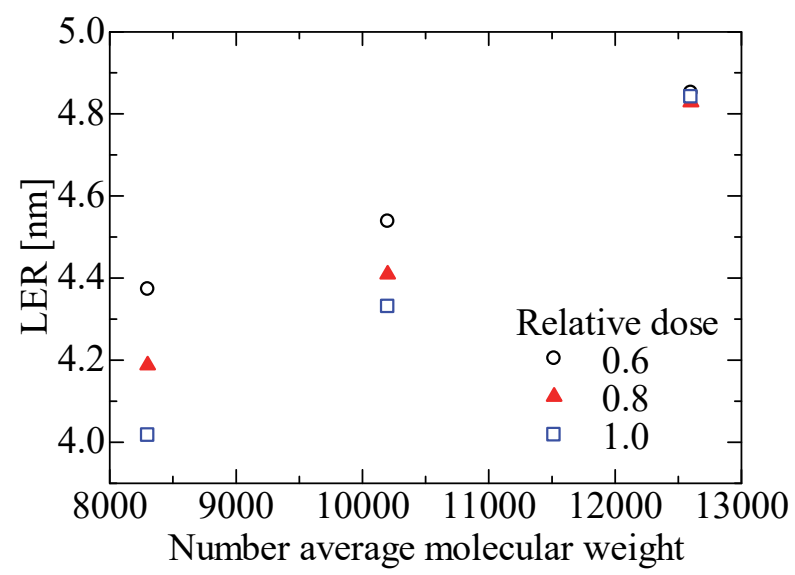

Fig. 7. Effect of $M_{\mathrm{n}}$ on the LER of the 14-nm-wide line pattern for three different doses.
The LER as a function of the PEB step number for various EUV light doses is shown in Fig. $8\left(M_{\mathrm{n}}\right.$ $=12600)$. With increasing PEB step number, the LERs first decrease and then increase. The LER reaches the minimum at smaller PEB step number with larger dose. These trends agree with the experimental trends in the optical lithography [18]. For each dose, there is an optimum PEB step number to minimize the LER of the pattern.

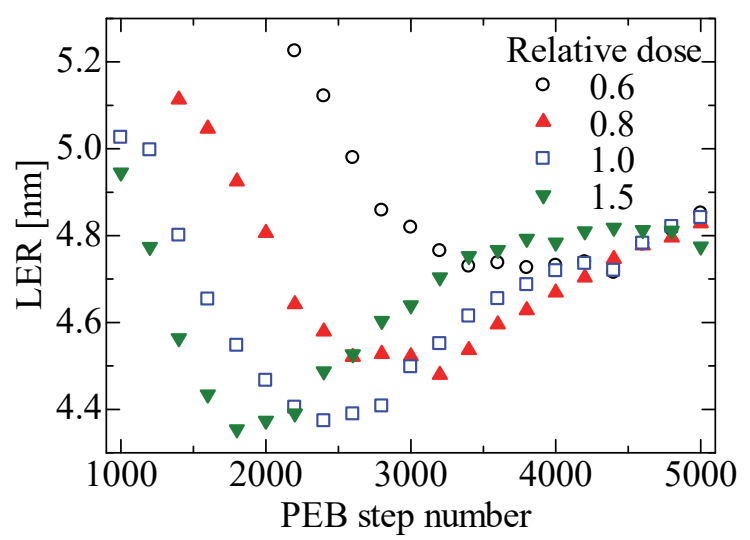

Fig. 8. Effect of the PEB step number on the LER of the 14 -nm-wide line pattern for various doses $\left(M_{\mathrm{n}}=12600\right)$.

The critical dimension (CD) error as a function of the PEB step number for various EUV light doses is shown in Fig. $9\left(M_{\mathrm{n}}=12600\right)$. With increasing PEB step number, the CD errors monotonically increase. The line width of the pattern becomes wider with larger dose. Because acid generation is greater for larger dose, the deprotection reaction proceeds earlier. This results in a wider pattern width for larger dose.

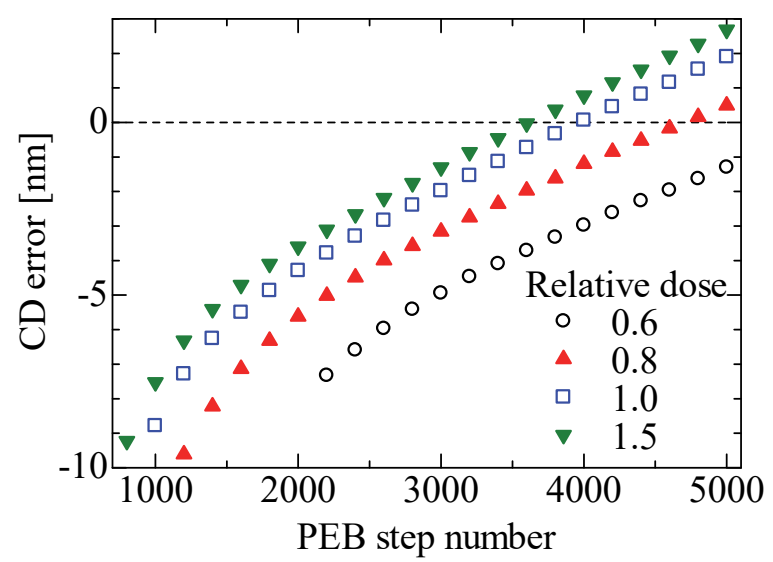

Fig. 9. Effect of the PEB step number on the CD error of the 14-nm-wide line pattern for various doses $\left(M_{\mathrm{n}}=\right.$ 12600).

\section{Conclusion}

A computational study of the pattern formation process for chemically amplified resists in EUV 
lithography based on the stochastic approach has been performed. The polymer molecule structure, acid diffusion, and polymer deprotection process were included in the simulation.

The fundamental properties, such as the molecular weight dependence and exposure condition effects, are well reproduced by the simulation. The LER of the pattern increases with increasing molecular weight of the resist. With increasing PEB step number, the LER first decreases and then increases. The LER reaches the minimum at smaller PEB step number with larger dose. The CD error monotonically increases with increasing PEB step number.

In the present study, we focused on introduction of the resist molecule structure into the simulation. Photoelectron scattering was roughly estimated. However, the shot noise effect [19] and secondary electron generation [20] greatly affect the pattern features. Improvement of the accuracy of the photoelectron and secondary electron scattering model is an important future subject to make the simulation useful for process and material design in EUV lithography.

\section{Acknowledgment}

We thank Edanz Group (www.edanzediting.com/ac) for editing a draft of this manuscript.

\section{References}

1. H. Kinoshita, K. Kurihara, Y. Ishii, and Y. Torii, $J$. Vac. Sci. Technol. B, 7 (1989) 1648.

2. C. W. Gwyn, R. Stulen, D. Sweeney, and D. Attwood, J. Vac. Sci. Technol. B, 16 (1998) 3142.

3. T. Kozawa, A. Saeki, and S. Tagawa, J. Vac. Sci. Technol. B, 22 (2004) 3489.

4. M. Kotera, K. Yagura, H. Tanaka, D. Kawano, and T. Maekawa, J. Vac. Sci. Technol. B, 47 (2008) 4944.

5. C. A. Mack, J. W. Thackeray, J. J. Biafore, and M.
D. Smith, J. Micro/Nanolith. MEMS MOEMS, 10 (2011) 033019.

6. T. Kozawa, Jpn. J. Appl. Phys., 52 (2013) 016501.

7. T. Kozawa, J. J. Santillan, and T. Itani, J. Photopolym. Sci. Technol., 31 (2018) 183.

8. M. Kim, J. Moon, J. Choi, S. Park, B. Lee, and M. Cho, Macromolecules, 51 (2018) 6922.

9. M. Koyama, M. Shirai, H. Kawata, Y. Hirai, and M. Yasuda, Jpn. J. Appl. Phys., 56 (2017) 06GL03.

10. M. Koyama, M. Shirai, H. Kawata, Y. Hirai, and M. Yasuda, J. Vac. Sci. Technol. B, 35 (2017) $06 \mathrm{G} 307$.

11. M. Yasuda, M. Koyama, R. Sakata, M. Shirai, H. Kawata, and Y. Hirai, J. Photopolym. Sci. Technol., 31 (2018) 189.

12. M. Yasuda, M. Koyama, M. Shirai, H. Kawata, and Y. Hirai, J. Vac. Sci. Technol. B, 36 (2018) 06JA04.

13. M. Koyama, M. Shirai, H. Kawata, Y. Hirai, and M. Yasuda, Jpn. J. Appl. Phys., 58 (2019) SDDB01.

14. H. Ito and C. G. Willson, Polym. Eng. Sci., 23 (1983) 1012.

15. L. F. Thompson, C. G. Willson, and M. J. Bowden, "Introduction to Microlithography", 2nd ed. (1994) 87.

16. F. M. Chapman. Jr. and L. L. Lohr. Jr., J. Am. Chem. Soc., 96 (1974) 4731.

17. M. D. Stewart, H. V. Tran, G. M. Schmid, T. B. Stachowiak, D. J. Becker, and C. G. Willson, J. Vac. Sci. Technol. B, 20 (2002) 2946.

18. T. Schnattinger and A. Erdmann, Proc. SPIE, 6923 (2008) 69230R.

19. M. Neisser, K. Y. Cho, C. Sarma, and K. Petrillo, J. Photopolym. Sci. Technol., 26 (2013) 617.

20. J. Torok, R. D. Re, H. Herbol, S. Das, I. Bocharova, A. Paolucci, L. E. Ocola, C. Ventrice Jr., E. Lifshin, G. Denbeaux, and R. L. Brainard, J. Photopolym. Sci. Technol., 26 (2013) 625. 